\title{
A Moving Boundary Wave Run-Up Model
}

\author{
Asu İnan ${ }^{1}$ and Lale Balas ${ }^{2}$ \\ Department of Civil Engineering, Faculty of Engineering and Architecture, \\ Gazi University, 06570 Ankara Turkey \\ asuinan@gazi.edu.tr, lalebal@gazi.edu.tr
}

\begin{abstract}
A numerical model has been developed for the simulation long wave propagation and run-up accounting for the bottom friction. Shallow water continuity and momentum equations are solved numerically by a two time step finite difference method. The upwind/downwind method is applied to the nonlinear advection terms and the continuity equation. Effects of damping and bottom friction on the computations are investigated. Since the equations lose their validity when waves break, wave breaking has been checked at every step of the computations. A point can be either wet or dry at different time levels. A moving boundary description and staggered grid are used to overcome the difficulty of determining wet and dry points. Equations are solved by the finite difference approximations of second and third order accuracy. Furthermore, space filters are applied to prevent parasitic short wave oscillations.
\end{abstract}

Keywords: Finite difference, long wave, run-up, moving boundary, filters.

\section{Introduction}

Nonbreaking long waves induced by tsunami, tide or storm cause catastrophic damages on the coasts because of high run-up levels. The numerical simulation of long wave propagation is an important tool in the damage control of catastrophic long waves. Estimation of the boundary between wet and dry points is a difficult problem in the simulation of wave run-up. During the simulations a computational point can be either wet or dry at different time levels. Therefore a moving boundary description is necessary.

Two dimensional nonlinear shallow water equations including bed shear stress were numerically solved by some researchers [1],[2]. Lynett et al. (2002) proposed a moving boundary technique to calculate wave run-up and run-down with depthintegrated equations [3]. An eddy viscosity model was inserted in the numerical model to account breaking phenomena. Kanoglu (2004) focused on initial value problem of the nonlinear evolution and run-up and run-down of long waves on sloping beaches for different initial wave forms [4]. Shermeneva and Shugan (2006) calculated the run-up of long gravity waves on a sloping beach using a high-order Boussinesq model [5]. Wei et al. (2006) simulated the long wave run-up under nonbreaking and breaking conditions with two dimensional well-balanced finite volume methods [6]. 
In this study, nonlinear long wave equations have been solved numerically to simulate the wave run-up by upwind-downwind method. The effects wave breaking, wetting-drying of boundary points, friction and nonlinear terms on wave run-up, have been investigated.

\section{Theory}

Continuity equation and equation of motion for long waves are given below where $\mathrm{x}$ is the propagation direction.

$$
\begin{gathered}
\frac{\partial u}{\partial t}+u \frac{\partial u}{\partial x}=-g \frac{\partial \eta}{\partial x}-\frac{r u|u|}{D} \\
\frac{\partial \eta}{\partial \mathrm{t}}=-\frac{\partial(\mathrm{Du})}{\partial \mathrm{x}}
\end{gathered}
$$

where, $\mathrm{u}, \mathrm{r}, \mathrm{D}, \mathrm{H}, \eta$ are horizontal velocity, bed friction factor, total depth, water depth and water elevation above still water level, respectively. Total depth is the sum of water depth and water elevation above the still water level.

Two-time level numerical scheme has been used for the solution of the system. Upwind/downwind method has been applied to the nonlinear (advective) terms. The following algorithm is used to check the wet and dry points. Total depth has a positive value at a wet point and it is zero on the boundary[7].

$$
\begin{array}{ll}
0.5\left(D_{j-1}+D_{j}\right) \geq 0 & \text { wet for point } D_{j} \\
0.5\left(D_{j-1}+D_{j}\right)<0 & \text { dry for point } D_{j}
\end{array}
$$

At every time level, wet and dry points are controlled and the validity of equation of motion has been provided. The equation of motion is modelled as given below;

$$
\frac{u_{j}^{t+1}-u_{j}^{t}}{\Delta t}+u_{p} \frac{\left(u_{j}^{t}-u_{j-1}^{t}\right)}{\Delta x}+u_{n} \frac{\left(u_{j+1}^{t}-u_{j}^{t}\right)}{\Delta x}=-g \frac{\left(\eta_{j}^{t}-\eta_{j-1}^{t}\right)}{\Delta x}+\frac{r u_{j}^{t}\left|u_{j}^{t}\right|}{0.5\left(D_{j}^{t}+D_{j-1}^{t}\right)}
$$

where, the $u_{p}$ and $u_{n}$ values are defined as;

$$
u_{p}=\frac{\left(u_{j}^{t}+\left|u_{j}^{t}\right|\right)}{2} ; \quad u_{n}=\frac{\left(u_{j}^{t}-\left|u_{j}^{t}\right|\right)}{2}
$$

Upwind/downwind approach has been applied to the continuity equation. Following scheme is applied to overcome the stability problem at the boundaries for wet and dry regions.

$$
\frac{\eta_{j}^{t+1}-\eta_{j}^{t}}{\Delta t}=-\frac{u_{p j 1} D_{j}^{t}+u_{n j 1} D_{j+1}^{t}-u_{p j} D_{j-1}^{t}-u_{n j} D_{j}^{t}}{\Delta x}
$$

where, $\mathrm{u}_{\mathrm{pj} 1}, \mathrm{u}_{\mathrm{pj}}, \mathrm{u}_{\mathrm{nj} 1}, \mathrm{u}_{\mathrm{nj}}$ are calculated as given below. 


$$
u_{p j 1}=\frac{\left(u_{j+1}^{t+1}+\left|u_{j+1}^{t+1}\right|\right)}{2} ; u_{n j 1}=\frac{\left(u_{j+1}^{t+1}-\left|u_{j+1}^{t+1}\right|\right)}{2} ; u_{p j}=\frac{\left(u_{j}^{t+1}+\left|u_{j}^{t+1}\right|\right)}{2} ; u_{n j}=\frac{\left(u_{j}^{t+1}-\left|u_{j}^{t+1}\right|\right)}{2}
$$

A staggered grid is used for spatial derivatives at wet and dry boundaries as shown in Fig. 1.

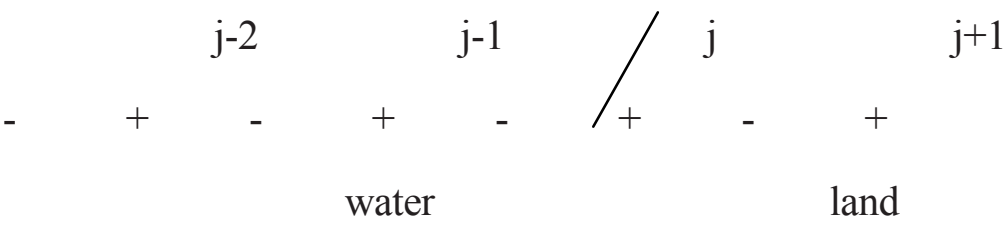

Fig. 1. Staggered grid

The intersection of wet and dry areas is a single point. The following extrapolation has been applied to the first dry point [8].

$$
\left(\eta_{j}^{t+1}\right)_{e x t}=2 \eta_{j-1}^{t+1}-\eta_{j-2}^{t+1} ; \quad\left(u_{j}^{t+1}\right)_{e x t}=2 u_{j-1}^{t+1}-u_{j-2}^{t+1}
$$

When waves break, the continuity equation and equation of motion lose their validity.

To check the breaking, a dimensionless parameter $B_{r}$ is used [9].

$$
B_{r}=\frac{\eta_{0} w^{2}}{g \alpha^{2}}
$$

where, $\eta_{0}$ is the initial wave amplitude, $w$ is angular frequency, $g$ is gravitational acceleration, $\alpha$ is bottom slope. If $\mathrm{B}_{\mathrm{r}}<1$, nonbreaking waves occur, otherwise the waves break while climbing up the sloping beach [2].

\section{Applications of the Model}

A sinusodial wave is assumed to enter from the left side of the channel with a water elevation function given below,

$$
\eta=\eta_{0} \sin \left(\frac{2 \Pi t}{T_{p}}\right)
$$

where $T_{p}$ is the wave period.

The application channel has a length of $2 \mathrm{~km}$. The bottom slope is linear. The water depth of the channel begins with $10 \mathrm{~m}$ at the left side of the channel and ends with $-10 \mathrm{~m}$ at the right side. Wave period is $1000 \mathrm{sec}$. The positive value of the depth descibes a wet region in the run-up computations. The horizontal grid size is $5 \mathrm{~m}$ in the propagation direction and the time step is $0.5 \mathrm{sec}$. [10]. 
Computations begin with describing a sinusodial signal at the left side of the channel. Then, the total depth is computed at each time level related according to the changes of water level with time. The boundary is described with $\mathrm{j}_{\mathrm{m}}$ index. If the water level at point $\mathrm{j}_{\mathrm{m}}$, is greater than the water depth, then, the point $\mathrm{j}_{\mathrm{m}+1}$ is considered in the calculations. After this control, velocity is calculated. Velocity at point $\mathrm{j}_{\mathrm{m}+1}$ is determined with the linear extrapolation and a similar approach is applied to the water elevation computation.

Computed water level changes at different levels of wave run-up and run- down is shown in Fig. 2. Bottom friction is neglected in this application. Curves 1 and 2 are the initial wave run-up curves, and curves 3 and 4 are the curves of wave run-down. Curve 5 shows the initiation of run- up of the second wave.

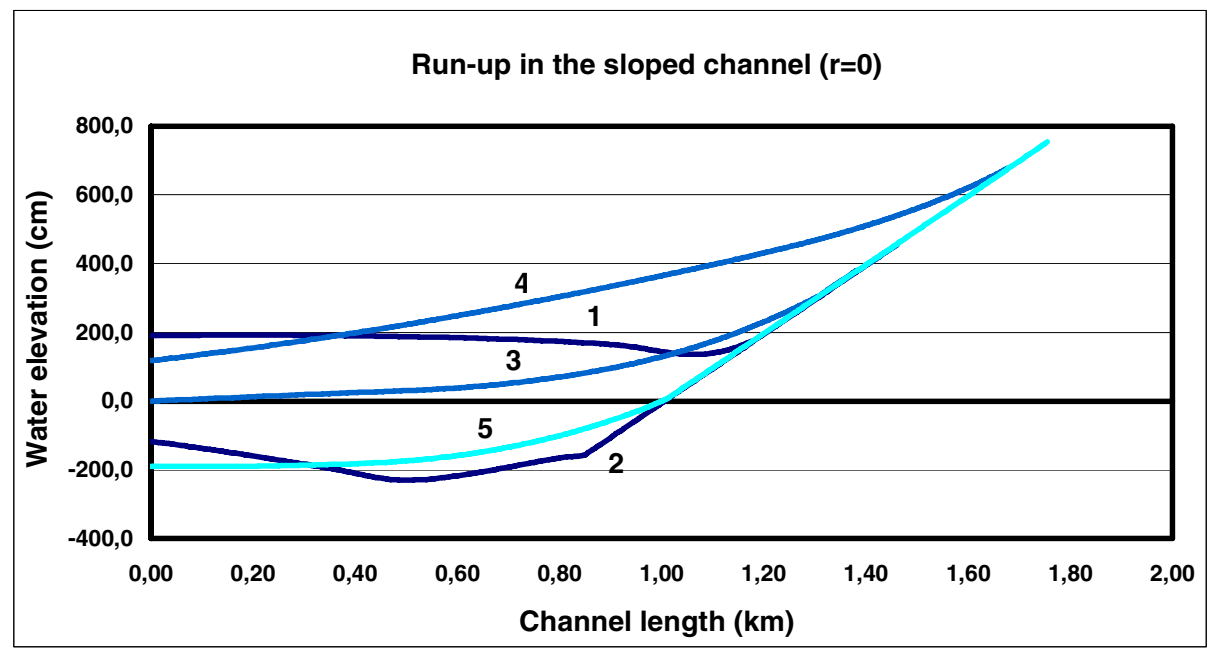

Fig. 2. Wave Run- up in the sloped channel

In the second application, bottom friction, nonlinear terms and damping effects are included in the computations while simulating the wave propagation along a channel with a positive slope. The length of the channel is $100 \mathrm{~km}$. Water depth varies linearly from $50 \mathrm{~m}$ to $5 \mathrm{~m}$. Long wave propagates from deep water to shallow water with a wave period of $T_{p}=5 \mathrm{~min}$. The time step is $0.1 \mathrm{sec}$ and the grid size is $25 \mathrm{~m}$. Simulated wave amplitude and velocity distributions throughout the channel are given in Fig.3.

Distributions of water elevation and velocity show a significant difference. Wave amplitudes change slightly along the channel, but velocities change significantly as shown in Fig.3. Damping is more donimant on wave amplitudes compared to velocities. Strong nonlinear interactions occur in shallow waters and they cause stability problems. To test whether the waves with small periods appeared at the beginning and at the end of the main wave are the results of a physical phenemenon 


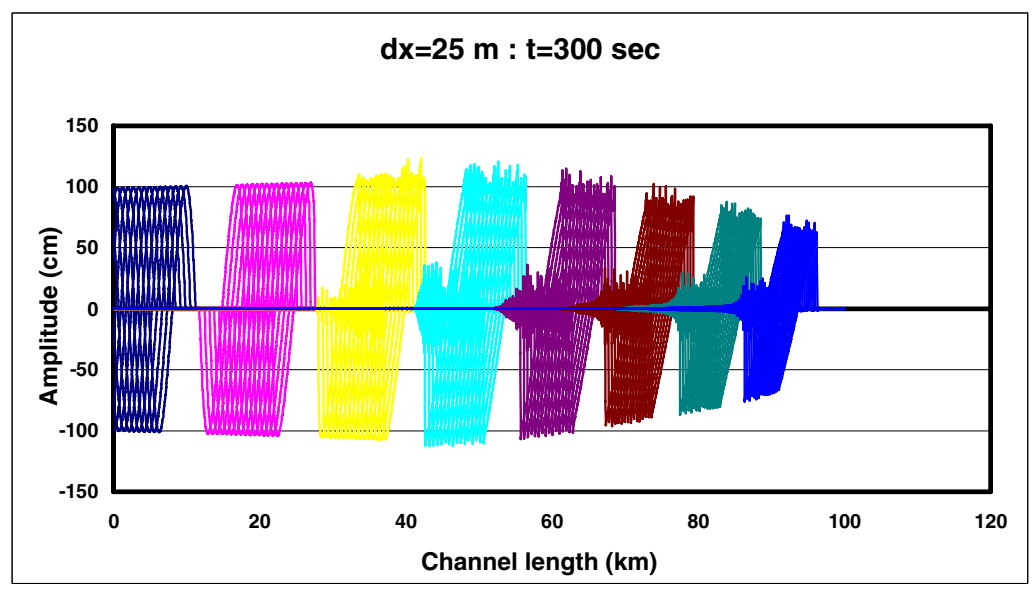

(a)

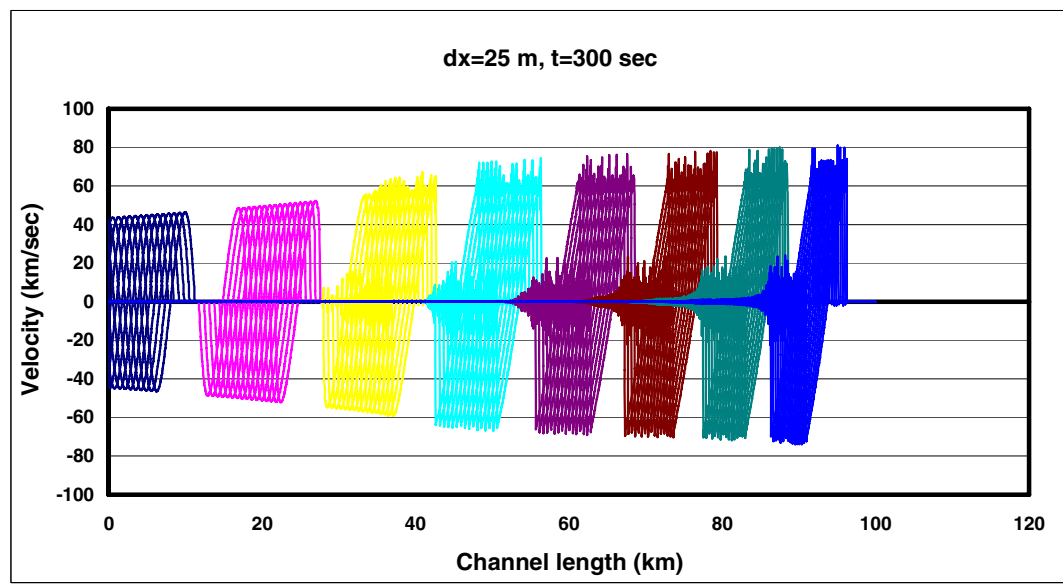

(b)

Fig. 3. a) Wave amplitude distribution b) Velocity distribution

or not, a finer resolution in the spatial domain has been used in the numerical computations. Therefore grid size is reduced to $5 \mathrm{~m}$ and the simulated wave amplitude changes are depicted in Fig. 4. Short wave oscillation decreases as resolution increases, so it can be concluded that short wave oscillations are the results of coarse spatial resolution.

The main sources of nonlinear effects are the advective terms. Because of stability problem, higher order accurate upwind approximations have been applied to first order derivatives. Three points are used in the solution scheme as;

$$
u \frac{\partial u}{\partial x} \approx u_{p} \frac{\left(3 u_{j}^{t}-4 u_{j-1}^{t}+u_{j-2}^{t}\right)}{2 h}+u_{n} \frac{\left(-u_{j+2}^{t}+4 u_{j+1}^{t}-3 u_{j}^{t}\right)}{2 h}+\theta\left(h^{3}\right)
$$




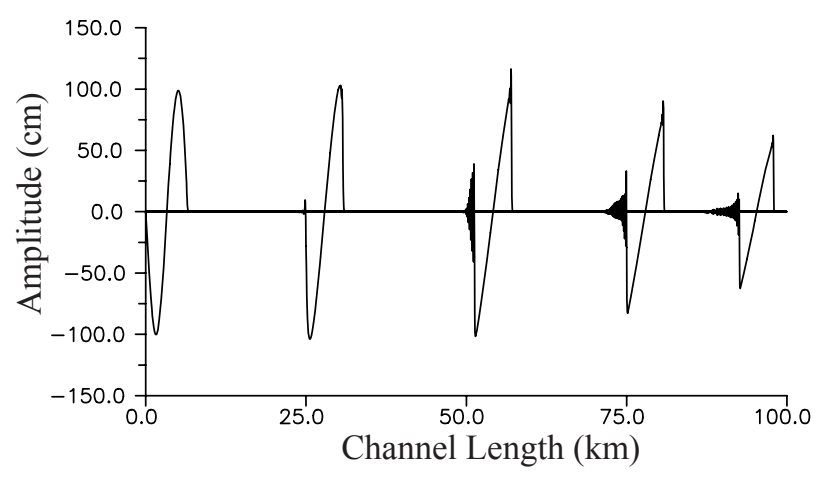

(a)

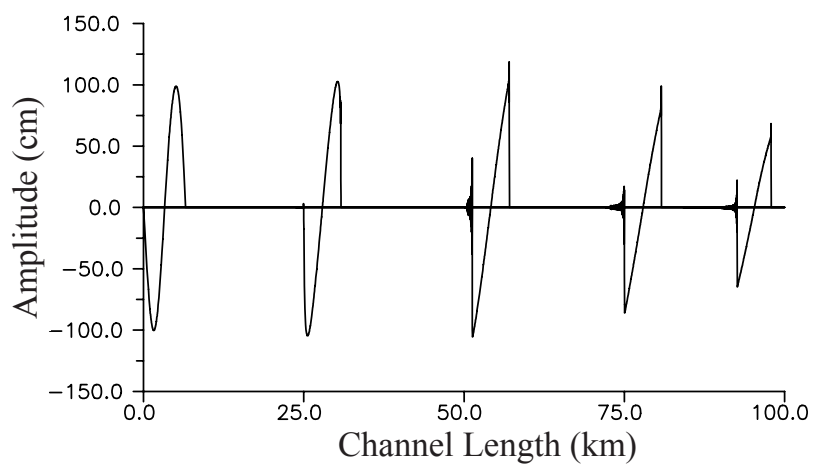

(b)

Fig. 4. Second order approximations a) $\Delta x=25 \mathrm{~m}, \Delta \mathrm{t}=0.1 \mathrm{sec}$ b) $\Delta \mathrm{x}=5 \mathrm{~m}, \Delta \mathrm{t}=0.1 \mathrm{sec}$

Higher order spatial approaches are used for the calculations of water elevations. The mesh point $\mathrm{j}$ is the point where velocity $\mathrm{u}_{\mathrm{j}}$ is calculated. Water elevation is calculated at $\Delta \mathrm{x} / 2$ distances away from point $\mathrm{j}$ at two sides. Higher order equations are obtained by combining the equations at points located at $\Delta x / 2$ and $3 \Delta x / 2$ distances and considering the second order Taylor series. The new formulation of the first derivative of water elevation is given below.

$$
\frac{\partial \eta}{\partial x}=\left[27\left(\eta_{j}-\eta_{j-1}\right)-\left(\eta_{j+1}-\eta_{j-2}\right)\right] / 24 \Delta x+\theta\left(h^{4}\right)
$$

A similar approach is used for the spatial derivative of velocity in the continuity equation. However, water elevation is computed at the midpoint in velocity computations and therefore spatial index is shifted to the right and point $j+1$ is inserted instead of point $\mathrm{j}$ in the numerical calculations.

$$
\left.\frac{\partial(H u)}{\partial x}=\left\{\begin{array}{l}
27\left[u_{j+1}\left(h_{j}+h_{j+1}\right) / 2-u_{j}\left(h_{j}+h_{j-1}\right) / 2\right] \\
u_{j+2}\left(h_{j+2}+h_{j+1}\right) / 2-u_{j-1}\left(h_{j-2}+h_{j-1}\right) / 2
\end{array}\right]\right\} / 24 \Delta x+\theta\left(h^{4}\right)
$$


Parasitic short wave oscillations are prevented with the application of higher order spatial and time resolutions, but application of simple space filters is easier to prevent the formation of parasitic short waves. Space filters are applied only to the computed velocities. The filtered new form of velocity is given in the equation (14).

$$
u_{n}(j)=u_{j}^{t+1}\left(1-\alpha^{*}\right)+0.25\left(u_{j-1}^{t+1}+2 u_{j}^{t+1}+u_{j+1}^{t+1}\right) \alpha^{*}
$$

where, $\alpha^{*}$ is a filter parameter and taken as 0.005 . The results obtained after the application of higher order approaches and filter technique are shown in Fig. 5.

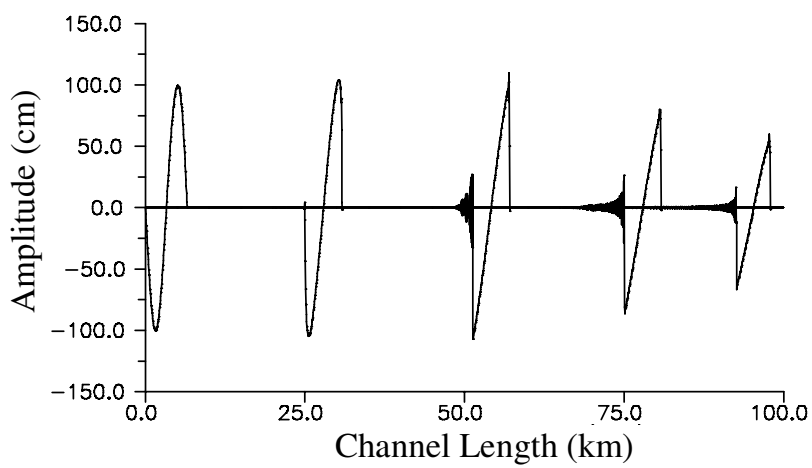

(a)

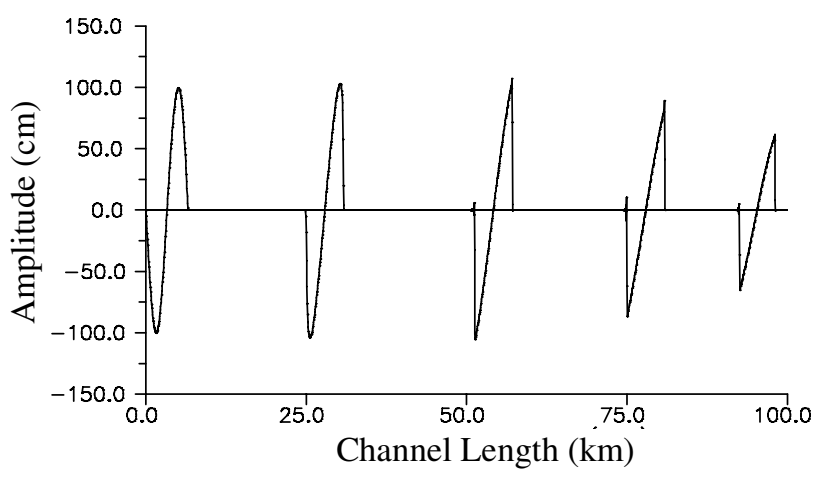

(b)

Fig. 5. a) Third order approximations b) Third order approximations and filter technique $(\Delta \mathrm{x}=25 \mathrm{~m}, \Delta \mathrm{t}=0.1 \mathrm{sec})$

\section{Conclusions}

Motion and continuity equations of long wave run-up are solved numerically by a two time level approach. The effects of nonlinear terms, damping, bottom friction and wet/ dry boundaries have been investigated. Application of upwind/downwind approximations 
to continuity equation gives stable results at the boundaries of wet and dry regions. Parasitic short wave oscillations are eliminated by increasing the resolutions in space and in time. Application of higher order accurate approximations increases the accuracy of the solution. It is observed that filter technique is a reliable tool for preventing parasitic short wave oscillations in the solutions obtained with lower order accurate approximations. The proposed numerical model successfully simulates the long wave run-up.

\section{References}

1. Hubbard, M.E., Dodd, N.:A 2D Numerical Model of Wave Runup and Overtopping. Coastal Engineering 47 (2002) 1-26.

2. Kowalik, Z.: Basic Relations between Tsunami Calculations and Their Physics. Science of Tsunami Hazard.19 (2001) 99-115.

3. Lynett, P. J., Wu, T-R., Liu P. L-F.:Modeling Wave Runup with Depth-Integrated Equations. Coastal Engineering, 46 (2002) 89-107.

4. Kanoglu, U.: Nonlinear Evolution and Runup- Rundown of Long Waves over a Sloping Beach. Journal of Fluid Mechanics, 513 (2004) 363-372.

5. Shermeneva, M.A., Shugan, I.V.: Calculating the wave runup on a low-sloping beach using a higher- order Boussinesq Model. Technical Physics Letter, 32 (2006) 64-66.

6. Wei, Y., Mao, X.Z., Cheung, K.F.: Well- Balanced Finite- Volume Model for Long- Wave Runup. Journal of Waterway, Port, Coastal and Ocean Engineering, ASCE, 132 (2006) 114-124.

7. Flather,R.A., Heaps, N.S. :Tidal computations for Morecamble Bay, Geophys. J. Roy. Astr. 42 (1975) 423-436.

8. Sielecki, A., Wurtele, M.G.: The numerical integration of the nonlinear shallow-water equations with sloping boundaries. J. Computational Physics 6 (1970) 219-236.

9. Voltzinger, N.E., Klevanny, K.A., Pelinovsky, E.N.: Long-Wave Dynamics of the Coastal Zone. Gidrometizdat., Leningrad (1989)

10. 10.Inan, A., Temiz, M., Balas, L.: Simulation of Long Wave Run-up, Proceedings of the V. National Conference on Turkish Coast'04, Adana, Turkey, 2 (2004) 891-898 